\title{
BMJ Open Fatty liver and mortality: a cohort population study in South Italy
}

\author{
Maria Gabriella Caruso, ${ }^{1,2}$ Nicola Veronese,${ }^{3}$ Maria Notarnicola, ${ }^{1}$ \\ Anna Maria Cisternino, ${ }^{2}$ Rosa Reddavide, ${ }^{2}$ Rosa Inguaggiato, ${ }^{2}$ Vito Guerra, ${ }^{4}$ \\ Rossella Donghia, ${ }^{4}$ Antonio Logroscino, ${ }^{5}$ Ornella Rotolo, ${ }^{2}$ Marisa Chiloiro, ${ }^{6}$ \\ Gioacchino Leandro, ${ }^{7}$ Giampiero De Leonardis, ${ }^{1,2}$ Valeria Tutino, ${ }^{1}$ G Misciagna, ${ }^{8}$ \\ Caterina Bonfiglio, ${ }^{9}$ Rocco Guerra, ${ }^{9}$ Alberto Osella ${ }^{9}$
}

To cite: Caruso MG, Veronese N, Notarnicola M, et al. Fatty liver and mortality: a cohort population study in South Italy. BMJ Open 2019;9:e027379. doi:10.1136/ bmjopen-2018-027379

- Prepublication history for this paper is available online. To view these files, please visit the journal online (http://dx.doi org/10.1136/bmjopen-2018027379).

Received 19 0ctober 2018 Revised 8 January 2019 Accepted 4 April 2019
Check for updates

(C) Author(s) (or their employer(s)) 2019. Re-use permitted under CC BY-NC. No commercial re-use. See rights and permissions. Published by BMJ.

For numbered affiliations see end of article.

Correspondence to Dr Maria Gabriella Caruso; gabriella.caruso@irccsdebellis.it

\section{ABSTRACT}

Objective Alcoholic fatty liver (AFLD) and non-alcoholic fatty liver (NAFLD) are two common conditions. However, if they can increase the risk of death is poorly explored. We therefore aimed to investigate the potential association between the presence and severity of liver steatosis and mortality in a large sample of older people.

Design Prospective.

Setting Community.

Participants Women and men randomly sampled from the electoral rolls of the population of Castellana Grotte, a town in Southern Italy (Apulia region) between 2005 and 2006. Among 1942 initially contacted, 1708 (=87.9\%) participated to the baseline survey (Multicentrica Colelitiasi III (MICOL III)). This specific study included 1445 older participants (mean age $=65.2$ years, females $=44.2 \%$ ).

Exposure NAFLD or AFLD.

Primary and secondary outcomes Mortality (all-cause and specific-cause).

Results After a median of 12 years, 312 participants $(=21.6 \%)$ died. After adjusting for nine potential confounders, the presence of steatosis was not associated with any increased risk of death in both NAFLD and AFLD. The severity of liver steatosis was not associated with any increased risk of mortality in NAFLD, while in AFLD, the presence of moderate steatosis significantly increased the risk of overall ( $\mathrm{HR}=2.16 ; 95 \% \mathrm{Cl} 1.19$ to 3.91$)$ and cancerspecific (HR=3.54; $95 \% \mathrm{Cl} 1.16$ to 10.87 ) death.

Conclusions Liver steatosis is not associated with any increased risk of death in NAFLD, while moderate steatosis could be a risk factor for mortality (particularly due to cancer) in people affected by AFLD.

\section{INTRODUCTION}

Fatty liver seems to be a common condition in Western countries, ${ }^{1}$ probably affecting about half of the individuals in some studies. ${ }^{2}$ It is estimated that nearly one in every three patients with persistently elevated alanine transaminase might have a fatty liver disease. ${ }^{3}$

Several risk factors have been recognised for the development of fatty liver. Among them, alcohol is probably the most important. Heavy alcohol intake is associated with fatty liver and afterwards with fibrosis, indicating

\section{Strengths and limitations of this study}

- The long follow-up (12 years) is a strength of our research.

- The use of liver echography for the diagnosis of liver steatosis (contrary to most studies using biohumoral parameters) is another strength of our work.

- The number of people with severe grade of either AFLD/NALFD is small and this can introduce an important bias in our findings.

- No liver biopsy (the gold standard method for the diagnosis of liver steatosis) is available.

- Comorbidities are self-reported and this could introduce another bias.

a form called alcoholic fatty liver (AFLD). ${ }^{4}$ In Western countries, however, fatty liver is usually associated with abdominal obesity and with metabolic syndrome features ${ }^{1}$ underlining a picture called non-alcoholic fatty liver (NAFLD). ${ }^{1}$

Even if the prevalence of the NAFLD varies according to the definition and the country, a recent systematic review and meta-analysis reported an overall prevalence of $25 \% .^{5}$ Independently from the origin, liver steatosis seems to be a significant predictor of several medical conditions including cardiovascular diseases (CVDs) ${ }^{6}$ type 2 diabetes $^{7}$ and liver cirrhosis. ${ }^{1}$ Metabolic conditions associated with NAFLD included obesity, present in more than half of the people, type 2 diabetes, hyperlipidemia, hypertension and metabolic syndrome. ${ }^{5}$ Liver-specific mortality and overall mortality in NAFLD patients is estimated in 0.77 persons per $1000 .^{5}$ However, the role of potential confounders seems to be important for explaining the association between the presence of liver steatosis and these medical conditions. For example, the association between liver steatosis and the onset of CVDs disappeared after the adjustment for potential confounders (eg, the 
presence of adiposity and diabetes) in some studies. ${ }^{8}$ Finally, because NAFLD is a heterogeneous disease, the advanced stages of which seem to be strongly affected by medical conditions (particularly insulin resistance and type 2 diabetes), further modifying the association between liver steatosis and mortality. ${ }^{9}$

Not univocal findings were reported regarding the presence of liver steatosis and mortality. Overall, it seems that a diagnosis of NAFLD is associated with a significant shorter survival than general population mainly due to higher rate of CVD. ${ }^{10-13}$ However, other studies reported that NAFLD was not associated with any increased risk of mortality. ${ }^{14}$ Moreover, many of the studies investigating the association between liver steatosis and mortality used elevated serum aminotransferases as a marker of NAFLD, which may underestimate the true prevalence of NAFLD of $>50 \% .{ }^{11}$ Finally, very limited data are available regarding the presence and severity of liver steatosis and mortality in people with AFLD.

Given this background, we aimed to investigate the potential association between the presence and severity of liver steatosis, diagnosed through liver echography and mortality in a large sample of older people living in a Mediterranean area.

\section{MATERIALS AND METHODS \\ Participants}

This study included women and men randomly sampled from the electoral rolls of the population of Castellana Grotte, a town in Southern Italy (Apulia region) between 2005 and 2006. Among 1942 initially contacted, 1708 $(=87.9 \%)$ participated to the baseline survey (Multicentrica Colelitiasi III (MICOL III)). No a priori selection criteria were used for the MICOL III study in terms comorbidities, while only people participating to the first wave in 1985-1986 and coming back 20 years after were included. The participants were enrolled through contact by mail, informing their general practitioners. For this specific research, being mortality the outcome of our interest, a trained researcher manually revised all the death certificates of the participants involved (other details are reported in the Outcome: vital status section).

Written informed consent, given during the first visit, was obtained from each participant before entering the study.

\section{Patient and public involvement}

Patients or public were not involved in the conception of the MICOL study.

\section{Measurements}

The survey visit consisted of the administration of a standardised questionnaire, including a validated semiquantitative food frequency questionnaire, anthropometric measurements, a blood sample for biochemical tests and an ultrasonographic examination. For the aims of this work, among the data regarding dietary habits, we reported data regarding alcohol intake and daily energy intake. A trained nurse recorded weight, height and waist circumference. The body mass index (BMI) was calculated and reported in $\mathrm{kg} / \mathrm{m}^{2}$. Blood pressure was also recorded measuring one time at the right arm of every participant by a trained nurse. Finally, self-reported information regarding smoking status (active or previous smoking during the first evaluation vs never), the presence of diabetes, gastric ulcer, cholecystic stones, hypertension and acute myocardial infarction, were used in the analyses.

\section{Exposure: fatty liver disease severity}

All the subjects underwent a standardised ultrasound examination made by two investigators using a Hitachi H21 Vision (Hitachi Medical Corporation, Tokyo, Japan). People with active chronic hepatitis (eg, HBV or HCV), acute forms, autoimmune hepatitis or haemochromatosis (based on self-reported information) were a priori excluded from this work. Examination of the visible liver parenchyma was performed with a $3.5 \mathrm{MHz}$ transducer. A scoring system was adopted to obtain a semiquantitative evaluation of fat in the liver. ${ }^{1516}$

The degree of liver fatty infiltration was graded according to the appearance of the liver echotexture, the liver echo penetration and the clarity of the liver blood vessels, as well as the liver diaphragm differentiation in echo amplitude. To each criterion, a score was assigned indicating the level of fatty liver infiltration. For each criterion, a score of 2 indicated a definite positive $(++)$ fatty liver infiltration, a score of 1 a probably positive $(+)$ fatty liver infiltration, a score of 0 the absence of fatty liver presence $(-)$. The fatty liver score ranged from 0 to 6 , higher values indicating higher severity. We therefore categorised the severity of liver steatosis in: absent $($ score $=0)$, mild $($ score $=1$ or 2$)$, moderate $($ score $=3$ or 4$)$ and severe (score $=5$ or 6 ). Liver steatosis was consequently divided in NAFLD or AFLD, using the standard cut-offs for alcoholic liver disease ( $30 \mathrm{~g}$ /day for men and $20 \mathrm{~g}$ / day for women). ${ }^{17}$ Alcohol intake was determined using the food frequency questionaire (FFQ), as stated before, using the intake of wine, beer and super-alcoholic drinks.

\section{Outcome: vital status}

The study's outcome of interest was all-cause mortality. In the MICOL III, mortality was confirmed and adjudicated death certificates manually revised by a trained researcher. The examination of the death certificates was done until 31 December 2017. Cardiovascular death was adjudicated through the codes of the International Classificiation Disease (ICD) 10 from I00 to I99 and cancer-related death with the codes from C00 to C97.

\section{Statistical analysis}

The analyses are shown by alcohol intake and by the presence or not of liver steatosis consequently having four groups: NAFLD (ie, liver steatosis with an alcohol intake $<30 \mathrm{~g} /$ day for men and $<20 \mathrm{~g} /$ day for women), the 
control group of people with NAFLD (ie, no presence of liver steatosis and low alcohol intake), AFLD (liver steatosis and an alcohol intake $\geq 30 \mathrm{~g}$ /day for men and $\geq 20 \mathrm{~g} /$ day for women) and the control group of AFLD (ie, high alcohol intake without any evidence of liver steatosis).

In each group, normal distributions of continuous variables were tested using the Kolmogorov-Smirnov test. Data are shown as mean \pm SD for quantitative measures, and percentages for all categorical variables. $\mathrm{P}$ values were calculated using the independent T-test for continuous variables and $\chi^{2}$ test for categorical ones.

Incidence rates are reported as number of deaths for 1000 persons-years. The proportional hazards assumption was checked by plotting the Schoenfeld residuals versus time without any violation and then Cox's regression analyses were performed. Using as exposure the presence of liver steatosis (NAFLD or AFLD), the basic model was not adjusted for any confounder; the fully adjusted model adjusted for: age, sex, smoking status (current/previous vs never), presence of diabetes, acute myocardial infarction (all yes vs no); waist circumference, systolic and diastolic blood pressure, daily energy (all as continuous). These covariates used for adjustment were factors significantly different between the presence or not of liver steatosis (considering a $\mathrm{p}<0.10$ ) or significantly associated with death according to univariate analysis (considering a $\mathrm{p}<0.20)$. The multicollinearity among covariates was also explored and taking a variance inflactory factor over 2 as criterion, the BMI was excluded having a high collinearity with waist circumference $(=4.68)$. We also ran the same analyses investigating the association between the severity of liver steatosis (categorised as absent, mild, moderate, severe) and mortality. In all the analyses, people with no liver steatosis were taken as reference and the Cox's regression analysis data are reported as HRs with $95 \%$ CIs. A similar analysis was run taking cardiovascular or cancer death as outcome and censoring people died for other causes or for cancer/cardiovascular death, respectively.

All the analyses were performed using SPSS V.17.0 for Windows. All statistical tests were two-tailed and statistical significance was assumed for a $\mathrm{p}<0.05$.

\section{RESULTS}

\section{Sample selection}

The MICOL III study initially included 1708 participants. After excluding 20 participants since they did not make the liver echography, 52 not having information regarding their alcohol intake, 17 with liver cirrhosis, 95 with a history of cancer and 79 without data regarding mortality status (lost at follow-up), we included 1445 participants in our analysis.

\section{General characteristics}

In the sample as whole, the mean age was $65.2 \pm 9.5$ years (range: $40-89$ ) and the women were the $44.2 \%$ of the whole population. The study subjects' characteristics by presence of liver steatosis and their alcohol intake are reported in table 1 .

Participants with liver steatosis and low alcohol intake (ie, NAFLD) $(n=457)$ did not differ in terms of age, percentage of females or smokers than those not having liver steatosis and low alcohol intake $(n=596)$. Even if the mean BMI was similar between the two groups $(\mathrm{p}=0.84)$, participants with NAFLD had a significant higher waist circumference and higher values in both systolic and diastolic blood pressure $(\mathrm{p}<0.0001$ for all comparisons). Conversely, no differences in terms of comorbidities were evident between the participants with NAFLD and those without (table 1).

People with AFLD (ie, high alcohol intake and liver steatosis) $(n=228)$ did not differ in terms of age, percentage of females, smoking habits, blood pressure or comorbidities than those with no AFLD $(n=164)$, even if the waist circumference was significantly higher in people with AFLD $(\mathrm{p}<0.0001)$ than those without (table 1$)$.

\section{Liver steatosis and mortality}

After a median period of 12 years, 312 participants (=21.6\% of the baseline population) died. The overall incidence was 19 (95\% CI 17 to 21) per 1000 personsyear. Of them, 95 died for cardiovascular conditions, 67 for cancer and 150 for other reasons.

Table 2 shows the association between liver steatosis and mortality by alcohol intake. After adjusting for 10 potential confounders at baseline (age, gender, demographics, comorbidities), the presence of steatosis was not associated with any increased risk of death for both NAFLD (HR=1.15; 95\% CI 0.87 to $1.51 ; \mathrm{p}=0.33)$ and AFLD $(\mathrm{HR}=1.24 ; 95 \% \mathrm{CI} 0.77$ to $2.01 ; \mathrm{p}=0.38)$. We further investigated whether the severity of liver steatosis was associated with higher mortality rate. After adjusting for potential confounders, while greater severity of liver steatosis was not associated with a higher risk of mortality in NAFLD, the presence of moderate liver steatosis increased the risk of overall death of more than two times $(\mathrm{HR}=2.16$; 95\% CI 1.19 to 3.91; $\mathrm{p}=0.01$ ) in AFLD (table 2).

In all the analyses, people without evidence of liver steatosis were taken as reference. Incidence rates are reported per 1000 persons-year with their 95\% CIs. The data are reported as HRs and 95\% CIs after adjusting for age, sex, smoking status (current/previous vs never), presence of diabetes, acute myocardial infarction (all yes vs no); waist circumference, systolic and diastolic blood pressure, daily energy (all as continuous).

Table 3 reports the data regarding the association liver steatosis and specific-cause mortality, namely death due to CVD or cancer, after adjusting for potential confounders. Again, while the presence and the severity of NAFLD were not associated with CVD or cancer death, the presence of moderate degree of liver steatosis significantly increased the risk of death related to cancer in AFLD (HR=3.54; $95 \%$ CI 1.16 to $10.87 ; \mathrm{p}=0.03$ ).

In all the analyses, people without evidence of liver steatosis were taken as reference. 
Table 1 Demographic and other characteristics of the study subjects by presence or not of liver steatosis and by alcohol intake

\begin{tabular}{|c|c|c|c|c|c|c|}
\hline & \multicolumn{3}{|l|}{ NAFLD* } & \multicolumn{3}{|l|}{ AFLD† } \\
\hline & No $(n=596)$ & Yes $(n=457)$ & P value $\neq$ & No $(n=228)$ & Yes $(n=164)$ & P value $\neq$ \\
\hline Age (years) & $65.3(9.8)$ & $66.3(9.6)$ & 0.11 & $63.6(8.6)$ & $64.1(8.6)$ & 0.53 \\
\hline Females (\%) & 54.4 & 57.6 & 0.30 & 14.1 & 13.0 & 0.78 \\
\hline $\begin{array}{l}\text { Smokers } \\
\text { (previous/actual) (\%) }\end{array}$ & 39.1 & 37.4 & 0.58 & 63.9 & 62.1 & 0.76 \\
\hline $\mathrm{BMI}\left(\mathrm{kg} / \mathrm{m}^{2}\right)$ & $29.9(5.4)$ & $29.9(5.8)$ & 0.84 & $29.3(5.1)$ & $29.4(4.7)$ & 0.95 \\
\hline Waist (cm) & $93.7(13.4)$ & $97.1(12.1)$ & $<0.0001$ & $96.3(12.2)$ & $100.1(11.4)$ & $<0.0001$ \\
\hline $\begin{array}{l}\text { Systolic blood pressure } \\
(\mathrm{mm} \mathrm{Hg})\end{array}$ & $120.9(20.9)$ & $127.9(19.0)$ & $<0.0001$ & $121.7(20.2)$ & $124.7(18.9)$ & 0.09 \\
\hline $\begin{array}{l}\text { Diastolic blood pressure } \\
(\mathrm{mm} \mathrm{Hg})\end{array}$ & $72.6(10.2)$ & $76.9(9.3)$ & $<0.0001$ & $73.9(9.6)$ & $75.8(12.9)$ & 0.12 \\
\hline Daily energy intake (kcal) & $2057(860)$ & $2068(782)$ & 0.83 & $2520(695)$ & $2592(801)$ & 0.33 \\
\hline Daily alcohol intake (g) & $6.6(7.6)$ & $6.9(7.4)$ & 0.49 & $51.7(21.3)$ & $50.3(20.8)$ & 0.51 \\
\hline Diabetes (\%) & 13.9 & 15.8 & 0.44 & 12.4 & 16.4 & 0.26 \\
\hline Hypertension (\%) & 50.2 & 56.3 & 0.05 & 39.8 & 45.5 & 0.27 \\
\hline Acute myocardial infarction (\%) & 4.9 & 4.6 & 0.89 & 4.1 & 6.3 & 0.37 \\
\hline
\end{tabular}

Values are reported as mean (SD) (for continuous variables), or \% for categorical ones.

${ }^{*}$ Alcohol consumption $<30 \mathrm{~g} /$ day (men), $<20 \mathrm{~g} /$ day (women).

$\dagger$ Alcohol consumption $\geq 30 \mathrm{~g} /$ day (men), $\geq 20 \mathrm{~g} /$ day (women).

$\ddagger P$ values were calculated using the independent T-test for continuous variables and $\chi^{2}$ test for categorical ones.

BMI, body mass index.

All the data are reported as HRs with their 95\% CIs, adjusted for age, sex, smoking status (current/previous vs never), presence of diabetes, acute myocardial infarction (all yes vs no); waist circumference, systolic and diastolic blood pressure, daily energy (all as continuous).

\section{DISCUSSION}

\section{Summary of findings}

In our study, during a median follow-up period of 12 years, we failed to find any significant association between the presence and severity of liver steatosis and mortality NAFLD, while, when limited to AFLD, the presence of moderate liver steatosis increased the risk of overall death of more than two times as well as this condition increased the risk of cancer-related death.

\section{Comparison with previous studies and novelty of our research} Overall, we have a limited knowledge if the presence of liver steatosis can increase per se the risk of mortality. One large study reported an increased risk of death among patients who had been admitted to the hospital and had a discharge diagnosis of fatty liver, but these findings are limited since the authors did not adjust for any potential confounder. ${ }^{18}$ On the contrary, two studies, including liver biopsy registries for identifying the presence of fatty liver disease found that the incidence of mortality was similar in those with fatty liver and general population. ${ }^{19} 20$ Another study following 144 patients with biopsy confirmed that NAFLD had similar survival probability than general population. ${ }^{21}$

However, all these studies explored the association between NAFLD and mortality in selected patients (eg, doing a biopsy for elevated liver enzymes or hospitalised), while NAFLD is highly prevalent in the general population. To our knowledge, only four cohort studies have evaluated the association between NAFLD and mortality in samples from the general population. ${ }^{14}$ 22-24 Three studies ${ }^{19-21}$ used liver enzyme levels as surrogate markers of NAFLD, consequently having an important bias in these findings and another one used an echographic method as we did. ${ }^{14}$ While the studies doing the diagnosis of NAFLD with raised liver enzyme levels found a higher risk of death in these people, the only study using the echography did not. Altogether, these finding probably suggest that only when liver has a certain grade of dysfunction can increase the risk of mortality. In this regard, one hypothesis can be the ability of the liver to regenerate in presence of steatosis given by the accumulation of triglycerides ${ }^{25}$ thus limiting the damage of liver and consequently not having any impact on mortality. Even if liver steatosis are followed by some important complications, it is estimated that only a few people will have them, that is, about one person over 10 having NAFLD will have a NASH and of them the incidence of liver cancer is estimated between $0 \%$ and $12 \% .{ }^{26}$ Only recently a large study made in 318224 people in Korea 


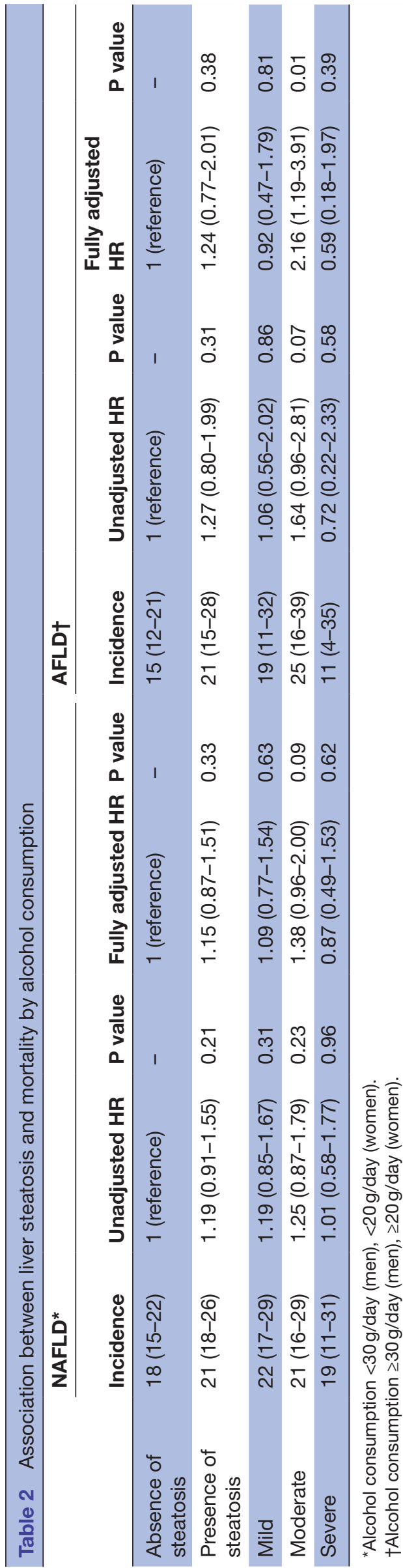

reported a significant association between NAFLD and mortality (all-cause and specific), but these findings were significant only in women and, surprisingly, in obese men, NAFLD was associated with a reduced risk of death from cancer. ${ }^{27}$ As correctly observed by Dr Stefan in an Editorial, it is likely that women involved in this study and having NAFLD clearly had a higher cardiometabolic risk profile, which might have increased the power to detect relationships of NAFLD with mortality. ${ }^{28}$

Finally, to the best of our knowledge, our study is the first exploring the association between liver steatosis and mortality in people having AFLD. Since the consumption of alcohol is overall increasing, ${ }^{29}$ the finding that the presence of moderate liver steatosis can significantly increase the risk of death (in particular for cancer) indicates that people having high alcohol intake should be routinely screened for liver steatosis in order to prevent the transition to moderate forms. We can probably justify the lack of the association between severe forms and death due to the limited number of people having this condition. Thus, other studies are necessary to confirm our findings.

\section{Limitations}

The findings of our study should be interpreted within its limitations. First, although liver biopsies and nuclear MRI are the methods of choice for diagnosis of liver steatosis, ultrasonography is currently the preferred method for screening asymptomatic patients with suspected liver injury. ${ }^{30}$ Several studies have demonstrated that the sensitivity, specificity and positive predictive value of ultrasound in detecting liver steatosis ranged widely $(60 \%-94 \%)$ depending on the population chosen for study. ${ }^{31}{ }^{32}$ Taking into account for the ethical concerns, we could not perform liver biopsy of our patients. Second, the number of people with severe grade of either AFLD/NALFD are small and this could affect our results. Third, contrary to our expectations, BMI was not different between people having or not NAFLD. We can hypothesise that, in our study, only abdominal obesity is strictly associated with the presence of NAFLD, while obesity per se no, further confirming a peculiar association between waist circumference and this condition. ${ }^{33}$ Fourth, we do not have information regarding liver enzymes and the calculation of markers of liver fibrosis (such as aspartate transaminases/alanino transaminase (AST/ALT) ratio or Fib-4) was not possible. Finally, the information regarding comorbidities are self-reported and this could introduce another bias such as we do not have any complete information regarding medications that, on the contrary, may significantly alter the association between liver steatosis and mortality. 
Table 3 Association between liver steatosis and specific-cause mortality by alcohol consumption

\begin{tabular}{|c|c|c|c|c|c|c|c|c|}
\hline & \multicolumn{4}{|l|}{ NAFLD* } & \multicolumn{4}{|l|}{ AFLD† } \\
\hline & CVD death & $P$ value & Cancer death & $P$ value & CVD death & $P$ value & Cancer death & $P$ value \\
\hline $\begin{array}{l}\text { Absence of } \\
\text { steatosis }\end{array}$ & 1 (reference) & - & 1 (reference) & - & 1 (reference) & - & 1 (reference) & - \\
\hline $\begin{array}{l}\text { Presence of } \\
\text { steatosis }\end{array}$ & $0.83(0.48-1.42)$ & 0.49 & $1.17(0.66-2.09)$ & 0.59 & $1.21(0.55-2.67)$ & 0.64 & $1.61(0.62-4.18)$ & 0.33 \\
\hline Mild & $0.47(0.19-1.11)$ & 0.09 & $1.24(0.61-2.50)$ & 0.55 & $0.97(0.31-3.04)$ & 0.96 & $0.67(0.14-3.24)$ & 0.62 \\
\hline Severe & $1.34(0.57-3.12)$ & 0.50 & $0.82(0.24-2.76)$ & 0.75 & Not possible & - & $1.06(0.13-8.83)$ & 0.96 \\
\hline
\end{tabular}

*Alcohol consumption $<30 \mathrm{~g} /$ day (men), $<20 \mathrm{~g} /$ day (women). †Alcohol consumption $\geq 30 \mathrm{~g} /$ day (men), $\geq 20 \mathrm{~g} /$ day (women).

CVD, cardiovascular disease.

\section{CONCLUSION}

Our study suggests that liver steatosis is not associated with any increased risk of death in NAFLD, while in AFLD moderate stages increased the risk of death, particularly for cancer-related death. Our findings suggest that other factors are probably more important in people with NAFLD for explaining their elevated rate of mortality, while a stricter ecographic follow-up should be proposed to people introducing high levels of alcohol. Since fatty liver disease is a common condition and continuously increasing, other longitudinal studies are needed to confirm/refute our findings.

\section{Author affiliations}

${ }^{1}$ Laboratory of Nutritional Biochemistry, National Institute of GastroenterologyResearch Hospital, IRCCS "Saverio de Bellis", Castellana Grotte, Italy

${ }^{2}$ Ambulatory of Clinical Nutrition, National Institute of Gastroenterology-Research Hospital, IRCCS "Saverio de Bellis", Castellana Grotte, Italy

${ }^{3}$ Istituto di Neuroscienze Consiglio Nazionale delle Ricerche, Padova, Italy ${ }^{4}$ Clinical Trial Unit, National Institute of Gastroenterology-Research Hospital, IRCCS "Saverio de Bellis", Castellana Grotte, Italy

"I.R.C.C.S. "Giovanni Paolo II", Thoracic Medical Oncology, Castellana Grotte, Italy ${ }^{6}$ Radiology Unit, National Institute for Digestive Disease, National Institute of Gastroenterology-Research Hospital, IRCCS "Saverio de Bellis", Castellana Grotte, Italy

${ }^{7}$ Gastroenterological Hospital, National Institute of Gastroenterology-Research Hospital, IRCCS "Saverio de Bellis", Castellana Grotte, Italy

${ }^{8}$ Epidemiology, National Institute of Gastroenterology-Research Hospital, IRCCS

"Saverio de Bellis", Castellana Grotte, Italy

${ }^{9}$ Laboratory of Biostatistics and Epidemiology, IRCCS "Saverio de Bellis", Castellana Grotte, Italy

Contributors MGC and NV designed the study and wrote the paper. MN, RD and VG interpreted the data. AMC, RR, RI, RG, OR, VT and GDL enrolled the subjects. AL, $\mathrm{CB}$ and $\mathrm{GL}$ analysed the data. MC performed biochemical analyses and ultrasound ecography. GM and $\mathrm{AO}$ conceived the study. GL and GM gave critical revisions to the final draft

Funding This work was supported by a Grant Ricerca Corrente 2018 n.25; Grant Ricerca Finalizzata Ministry of Health, years 2004-2006 (N ICS-160.2/RF03.111).

Competing interests None declared.

Patient consent for publication Not required.

Ethics approval The proposal of this research was approved by the Institutional Review Board (Ethical Committee) of IRCCS De Bellis.

Provenance and peer review Not commissioned; externally peer reviewed.

Data sharing statement Technical appendix, statistical code, and datasets are available from request to the corresponding author, upon reasonable request. The data are deidentified and they are available, upon reasonable request, to Nicola Veronese and Maria Gabriella Caruso, under the written permission of the Institute.

Open access This is an open access article distributed in accordance with the Creative Commons Attribution Non Commercial (CC BY-NC 4.0) license, which permits others to distribute, remix, adapt, build upon this work non-commercially, and license their derivative works on different terms, provided the original work is properly cited, appropriate credit is given, any changes made indicated, and the use is non-commercial. See: http://creativecommons.org/licenses/by-nc/4.0/.

\section{REFERENCES}

1. Bedogni G, Nobili V, Tiribelli C. Epidemiology of fatty liver: An update. World Journal of Gastroenterology: WJG 2014;20:9050-4.

2. Bedogni G, Miglioli L, Masutti F, et al. Prevalence of and risk factors for nonalcoholic fatty liver disease: the Dionysos nutrition and liver study. Hepatology 2005;42:44-52.

3. Armstrong MJ, Houlihan DD, Bentham L, et al. Presence and severity of non-alcoholic fatty liver disease in a large prospective primary care cohort. J Hepatol 2012;56:234-40.

4. Lieber CS. Alcoholic fatty liver: its pathogenesis and mechanism of progression to inflammation and fibrosis. Alcohol 2004;34:9-19.

5. Younossi ZM, Koenig AB, Abdelatif D, et al. Global epidemiology of nonalcoholic fatty liver disease-Meta-analytic assessment of prevalence, incidence, and outcomes. Hepatology 2016;64:73-84.

6. Mellinger JL, Pencina KM, Massaro JM, et al. Hepatic steatosis and cardiovascular disease outcomes: An analysis of the Framingham Heart Study. J Hepatol 2015;63:470-6.

7. Roden M. Mechanisms of Disease: hepatic steatosis in type 2 diabetes--pathogenesis and clinical relevance. Nat Clin Pract Endocrinol Metab 2006;2:335-48.

8. Pickhardt PJ, Hahn L, Muñoz del Rio A, et al. Natural history of hepatic steatosis: observed outcomes for subsequent liver and cardiovascular complications. AJR Am J Roentgenol 2014;202:752-8.

9. Stefan N, Häring HU, Cusi K. Non-alcoholic fatty liver disease: causes, diagnosis, cardiometabolic consequences, and treatment strategies. Lancet Diabetes Endocrinol 2019;7.

10. Adams LA, Lymp JF, St Sauver J, et al. The natural history of nonalcoholic fatty liver disease: a population-based cohort study. Gastroenterology 2005;129:113-21.

11. Bellentani S, Marino M. Epidemiology and natural history of nonalcoholic fatty liver disease (NAFLD). Ann Hepatol 2009;8(Suppl 1):S4-8.

12. Xun YH, Guo JC, Lou GQ, et al. Non-alcoholic fatty liver disease (NAFLD) fibrosis score predicts 6.6-year overall mortality of Chinese patients with NAFLD. Clin Exp Pharmacol Physiol 2014;41:643-9.

13. Söderberg C, Stål $P$, Askling J, et al. Decreased survival of subjects with elevated liver function tests during a 28 -year follow-up. Hepatology 2010;51:595-602.

14. Lazo M, Hernaez R, Bonekamp S, et al. Non-alcoholic fatty liver disease and mortality among US adults: prospective cohort study. BMJ 2011;343:d6891.

15. Chiloiro M, Caruso MG, Cisternino AM, et al. Ultrasound evaluation and correlates of fatty liver disease: a population study in a Mediterranean area. Metab Syndr Relat Disord 2013;11:349-58. 
16. Chiloiro M, Riezzo G, Chiarappa S, et al. Relationship among fatty liver, adipose tissue distribution and metabolic profile in moderately obese children: an ultrasonographic study. Curr Pharm Des 2008:14:2693-8.

17. O'Shea RS, Dasarathy S, McCullough AJ. Practice Guideline Committee of the American Association for the Study of Liver DiseasesPractice Parameters Committee of the American College of Gastroenterology. Alcoholic liver disease. Hepatology 2010;51:307-28.

18. Jepsen $\mathrm{P}$, Vilstrup $\mathrm{H}$, Mellemkjaer L, et al. Prognosis of patients with a diagnosis of fatty liver--a registry-based cohort study. Hepatogastroenterology 2003;50:2101-4.

19. Dam-Larsen S, Franzmann M, Andersen IB, et al. Long term prognosis of fatty liver: risk of chronic liver disease and death. Gut 2004:53:750-755.

20. Teli MR, James OF, Burt AD, et al. The natural history of nonalcoholic fatty liver: a follow-up study. Hepatology 1995;22:1714-9.

21. Ekstedt M, Franzén LE, Mathiesen UL, et al. Long-term follow-up of patients with NAFLD and elevated liver enzymes. Hepatology 2006;44:865-73.

22. Ruhl CE, Everhart JE. Elevated serum alanine aminotransferase and gamma-glutamyltransferase and mortality in the United States population. Gastroenterology 2009;136:477-85.

23. Dunn W, Xu R, Wingard DL, et al. Suspected nonalcoholic fatty liver disease and mortality risk in a population-based cohort study. Am J Gastroenterol 2008;103:2263-71.

24. Ong JP, Pitts A, Younossi ZM. Increased overall mortality and liver-related mortality in non-alcoholic fatty liver disease. $J$ Hepatol 2008;49:608-12.
25. Choi SS, Diehl AM. Hepatic triglyceride synthesis and nonalcoholic fatty liver disease. Curr Opin Lipidol 2008;19:295-300.

26. Bhala N, Younes R, Bugianesi E. Epidemiology and natural history of patients with NAFLD. Curr Pharm Des 2013;19:5169-76.

27. Hwang YC, Ahn HY, Park SW, et al. Nonalcoholic fatty liver disease associates with increased overall mortality and death from cancer, cardiovascular disease, and liver disease in women but not men. Clin Gastroenterol Hepatol 2018;16:1131-7.

28. Stefan N. Nonalcoholic fatty liver disease and mortality. : Elsevier, 2018:16: 1043-5.

29. Mills SJ, Harrison SA. Comparison of the natural history of alcoholic and nonalcoholic fatty liver disease. Curr Gastroenterol Rep 2005;7:32-6.

30. Castera L, Vilgrain V, Angulo P. Noninvasive evaluation of NAFLD. Nat Rev Gastroenterol Hepatol 2013;10:666-75.

31. Liang RJ, Wang HH, Lee WJ, et al. Diagnostic value of ultrasonographic examination for nonalcoholic steatohepatitis in morbidly obese patients undergoing laparoscopic bariatric surgery. Obes Surg 2007;17:45-56.

32. Hamaguchi M, Kojima T, Itoh $\mathrm{Y}$, et al. The severity of ultrasonographic findings in nonalcoholic fatty liver disease reflects the metabolic syndrome and visceral fat accumulation. Am J Gastroenterol 2007:102:2708-15.

33. Pang Q, Zhang JY, Song SD, et al. Central obesity and nonalcoholic fatty liver disease risk after adjusting for body mass index. World $J$ Gastroenterol 2015;21:1650. 